\title{
Cellulites cervico-faciales : à propos de 41 cas
}

\section{Cervicofacial cellulitis: report of 41 cases}

\author{
RA RAKOTOARISON, NP RAMAROZATOVO, FA RAKOTO, FJ RAKOTOVAO
}

\section{RÉSUMÉ}

La cellulite cervico-faciale est une infection polymicrobienne du tissu cellulo-adipeux ayant une tendance extensive. La médiastinite en constitue la complication la plus redoutée.

Cette étude a pour objectifs de rappeler le risque lié à la négligence d'une carie dentaire, de mettre en garde contre l'utilisation des anti-inflammatoires et de rappeler l'importance d'une prise en charge précoce et adaptée. II s'agit d'une étude rétrospective portant sur 41 cas pris en charge au Centre Hospitalier de SoavinandrianaAntananarivo (CENHOSOA) de 1997 à 2003.

C'est une pathologie infectieuse dont la fréquence augmente chaque année. Un déficit immunitaire est souvent suspecté. Elle touche surtout le sujet jeune et le sexe masculin. La porte d'entrée la plus fréquente est une carie dentaire négligée. Le germe responsable est le plus souvent un anaérobie (staphylocoque aureus, streptocoques). L'utilisation isolée d'anti-inflammatoire semble être un facteur favorisant la prolifération et la virulence des germes. Le traitement doit être précoce et il est basé sur la perfusion d'antibiotique adéquate, à forte dose. Une incision-drainage est nécessaire en cas de collection purulente. Le pronostic est généralement bon, mais l'affection peut être fatale en cas de négligence ou de traitement insuffisant par extension rapide vers le médiastin. Med Buccale Chir Buccale 2007; 13 : 35-39.

mots clés: cellulite, carie dentaire, traitement anti-inflammatoire, antibiothérapie, médiastinite.

médecine buccale chirurgie buccale

VOL. $14, \mathrm{~N}^{\circ} 1$ 2008 page 35

\section{SUMMARY}

Cervicofacial cellulitis is a soft tissue infection that tends to extension. It is due to polygerme. Mediastinitis is the most serious complication.

The aims of this study are to remind the bad effect of neglected teeth decay, to pay attention of the use of antiinflammatory and to remind the importance of the early treatment. It is a retrospective study about 41 cases wich are taken care in the Soavinandriana Hospital Center (CENHOSOA) in Antananarivo from 1997 to 2003.

It is an infectious pathology with yearly increase frequency. The reduction of the immune defense is related. It mainly affects young person with male predominance. Tooth decay is the main etiology. The germs are especially anaerobia (staphylococcus aureus, streptococcus). Anti-inflammatory makes the germs virulent and prolifered. The treatment must be started early and it is based on high dose of right antibiotic. An incision is necessary if pus is formed. Usually, it is a good prognostic pathology, but it can be serious if there is late or bad treatment. Med Buccale Chir Buccale 2007; 13: 35-39.

key words: cellulitis, tooth decay, anti-inflammatory drug, antibiotherapy, mediastinitis. 
La cellulite cervico-faciale est une complication des infections d'origine dentaire négligées. Son évolution peut engager le pronostic vital par son extension vers le médiastin : la médiastinite s'accompagne d'une inondation purulente des poumons provoquant une dyspnée qui complique la prise en charge et assombrit le pronostic.

Epidémiologiquement, les cellulites cervico-faciales sont des affections rares du sujet jeune, surtout masculin [1]. L'étiologie la plus fréquente est la carie dentaire. Au CENHOSOA Antananarivo, elles représentent 1,60\% des hospitalisations dans le service d'Oto-Rhino-Laryngologie et de Chirurgie Cervico-Maxillo-Faciale et $48,38 \%$ sont d'origine dentaire.

\section{PATIENTS ET MÉTHODE}

médecine buccale chirurgie buccale

VOL. $14, \mathrm{~N}^{\circ} 1$ 2008

page 36
II s'agit d'une étude rétrospective portant sur 41 patients âgés de 4 à 63 ans, pris en charge entre 1997 et 2003. Elle est basée sur l'examen du registre des entrées et des sorties et sur l'examen de dossiers des patients (fiches de consultation, fiches d'examens complémentaires, fiches de traitement et de suivi).

Les patients sélectionnés sont ceux qui ont été hospitalisés et présentant un tableau de cellulite faciale et/ou cervico-faciale quelle que soit leur étiologie. Les patients non hospitalisés (vus seulement en consultation externe), et ceux dont les dossiers sont incomplets ont été exclus de l'étude.

Les paramètres étudiés sont la fréquence, l'âge, le sexe, la porte d'entrée, les facteurs favorisants, la bactériologie, le traitement et l'évolution.

\section{RÉSULTATS}

Ils sont présentés sous forme de tableaux synoptiques. La valeur des pourcentages a été arrondie à l'unité la plus proche.
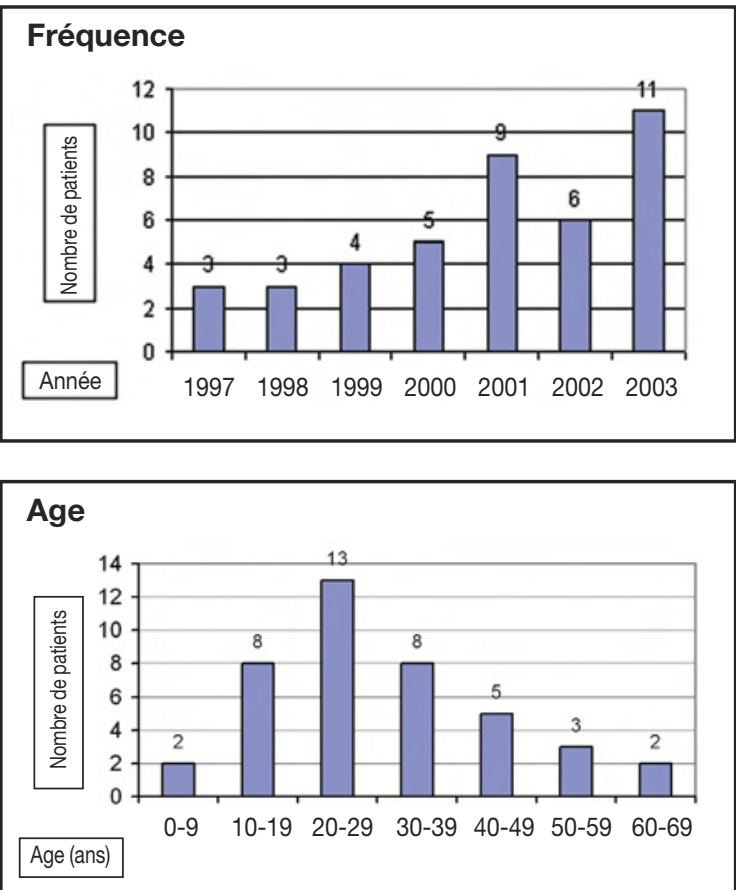

Sexe

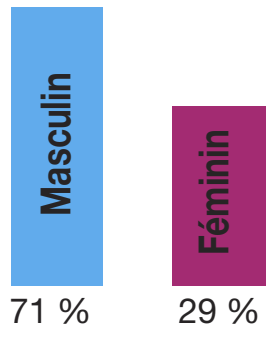

\begin{tabular}{lcc|}
\hline Porte d'entrée & & \\
& Nombre de cas & Pourcentage \\
\hline Dentaire & 30 & $74 \%$ \\
\hline Pharyngée & 2 & $5 \%$ \\
Sinus maxillaire & 2 & $5 \%$ \\
\hline Cutanée & 2 & $5 \%$ \\
\hline Auriculaire & 1 & $2 \%$ \\
Thyroïdienne & 1 & $2 \%$ \\
Traumatique & 1 & $2 \%$ \\
\hline Non précisée & 2 & $5 \%$ \\
\hline
\end{tabular}




\begin{tabular}{|lcc|}
\hline \multicolumn{2}{|c|}{ Facteurs favorisants } & \\
\hline Anti-inflammatoires & 21 & Pourcentage \\
\hline Alcool - tabac & 4 & $52 \%$ \\
\hline Diabète & 1 & $10 \%$ \\
Grossesse & 1 & $2 \%$ \\
\hline Dénutrition & 1 & $2 \%$ \\
\hline Inconnus & 13 & $2 \%$ \\
\hline
\end{tabular}

\section{Examens bactériologiques}

\begin{tabular}{cc} 
Nombre de cas & Germes identifiés \\
44 & Staphylocoques aureus \\
4 & Streptocoques \\
7 & Prélèvement stérile \\
\hline
\end{tabular}

\section{Evolution}

\begin{tabular}{lcc} 
& Nombre de cas & Pourcentage \\
\hline Favorable & 38 & $93 \%$ \\
\hline Récidive & 2 & $5 \%$ \\
\hline Décès & 1 & $2 \%$ \\
\hline
\end{tabular}

\section{COMMENTAIRES}

\section{La fréquence}

L'augmentation annuelle de façon presque régulière, du nombre de cas de cellulites cervicofaciales semble due à une diminution de la défense immunitaire causée par une automédication abusive à base d'anti-inflammatoires, au lieu d'utiliser des antalgiques non anti-inflammatoires ${ }^{[2]}$. De plus, la mauvaise utilisation des antibiotiques, toujours en automédication, provoque une augmentation de la résistance et de la virulence des germes. Cette augmentation du nombre de cas de cellulites est également relatée par Potard dans une étude portant sur 13 cas ${ }^{[3]}$.

\section{Le sexe}

Comme plusieurs auteurs ${ }^{[2-3-4]}$, on observe une nette prédominance du sexe masculin $(71 \%$ des cas). Une meilleure réponse immunitaire chez la femme est évoquée dans différents articles. Cependant, une étude effectuée par Kpemissi en 1989 au Togo sur 26 patients a donné une prédominance du sexe féminin ; mais le nombre insuffisant de cas ne permet pas de confirmer avec certitude cette hypothèse ${ }^{[4]}$.

\section{L'âge ${ }^{[2-4]}$}

Cette affection peut toucher des sujets de tout âge, mais elle intéresse surtout les sujets jeunes. L'âge moyen de nos observations est de 30,36 ans avec un pic dans la tranche d'âge comprise entre 20 et 29 ans. Dans les études de Barnabe et de Potard, l'âge moyen est respectivement de 56 et 51 ans, alors qu'il est de 28 ans dans celle de Kpemissi. Cela pourrait expliquer une éventuelle relation entre la race et la cellulite; elle atteint surtout les sujets jeunes dans la race noire et les sujets âgés dans la race blanche.

\section{La porte d'entrée}

Les cellulites cervico-faciales sont d'origine très variée mais la plupart sont d'origine dentaire et pharyngée [5]. Toutes les dents peuvent être à l'origine d'une cellulite mais, le plus souvent, ce sont les molaires inférieures qui sont responsables. La pathologie initiale est une nécrose pulpaire évoluant vers une infection péri-apicale, une péricoronarite de la dent de sagesse ou une affection parodontale [6].

- L'infection péri-apicale, secondaire à une nécrose pulpaire est la cause la plus fréquente. Elle peut être le résultat d'une infection péri-apicale aiguë d'emblée ou d'une infection chronique « réchauffée ».

- Dans la péricoronarite sur dent de sagesse, l'infection du capuchon muqueux peut gagner rapidement le tissu cellulo-graisseux du voisinage.

- En fonction de la profondeur de la poche parodontale et du siège de l'ensemencement microbien, l'infection peut évoluer vers un abcès sous la fibromuqueuse gingivale ou vers une cellulite. D'autres portes d'entrée ont été rapportées: phlegmon péri-amygdalien, lithiase submandibulaire, parotidite, cellulite post-chirurgicale, sinusite maxillaire, otite moyenne chronique, cellulite thyroïdienne, surinfection d'un kyste du tractus thyréoglosse, voire cellulite secondaire à un corps étranger (arête de poisson....). ${ }^{7-8]}$ médecine

buccale chirurgie buccale

VOL. $14, \mathrm{~N}^{\circ} 1$ 2008

page 37 
médecine

buccale

chirurgie

buccale

VOL. $14, \mathrm{~N}^{\circ} 1$ 2008

page 38
En raison de la gravité, l'infection se propage habituellement vers le bas, par extension directe de proche en proche, par voie lymphatique ou hématogène. L'atteinte médiastinale est le résultat d'une " coulée de cellulite » le long des aponévroses cervicales à travers les trois principaux espaces anatomiques: l'espace rétroviscéral, la gouttière vasculaire et l'espace prétrachéal $[2,9,10]$.

\section{Les facteurs favorisants}

Les anti-inflammatoires non stéroïdiens (AINS) représentent la classe thérapeutique la plus consommée dans le monde [11]. Ils sont souvent considérés comme les principaux facteurs favorisant des cellulites cervico-faciales. Ils sont en général pris seuls ou associés à une antibiothérapie le plus souvent inadaptée [10-12]. La notion d'une prise d'AINS est retrouvée dans plus de la moitié de nos cas (52\%).

L'inflammation résulte d'un ensemble de phénomènes de défense humorale et cellulaire survenant devant une agression exogène ou endogène. Elle est donc habituellement bénéfique, mais elle peut être néfaste du fait de sa localisation ou de certains dérèglements ${ }^{[11]}$. En diminuant les premiers signes de l'inflammation, ils peuvent retarder la première consultation. Les AINS semblent augmenter la virulence et la prolifération microbienne dans les gangrènes streptococciques [13]. SIMON et al. recommandent l'interdiction de leur prescription dans le traitement des cellulites [14]. Les corticoïdes sont également considérés comme des facteurs favorisant la diffusion des cellulites. Ils ont une action immunosuppressive sur le mécanisme humoral de défense. Elles réduisent de façon significative la synthèse des immunoglobulines $G$ et inhibent l'adhérence des polynucléaires et la capacité phagocytaire des macrophages [15-16].

Outre les anti-inflammatoires, le diabète et l'antibiothérapie inadaptée (molécule ou posologie) ${ }^{[3]}$ sont souvent évoqués comme facteurs favorisants des cellulites. Les autres facteurs souvent cités sont l'alcool, le tabac, la toxicomanie, la grossesse, une hémopathie sous chimiothérapie, une aplasie médullaire, la malnutrition, l'insuffisance rénale chronique.

\section{Les germes responsables}

II s'agit d'une infection polymicrobienne. Les germes les plus fréquemment présents sont des germes anaérobies. On distingue les staphylocoques, les streptocoques, le genre Clostridium (perfringens et histolyticum), les fusiformes et les actinomyces [17-18]. Ce sont des germes commensaux et saprophytes de la cavité buccale, localisés principalement dans les sillons dentaires et gingivaux [3-6].

Sur 15 prélèvements de pus effectués, les examens bactériologiques ont identifié du staphylocoque aureus dans 4 cas (cellulites d'origine cutanée), des streptocoques dans 4 cas ; dans 7 cas le prélèvement était stérile. Dans les cellulites d'origine dentaire, les germes en cause sont très souvent les streptocoques. Une antibiothérapie instaurée avant le prélèvement donnerait un faux résultat à l'examen bactériologique. De plus, le conditionnement du prélèvement doit respecter un environnement favorable aux germes (température, anaérobie). Une hémoculture en période d'hyperthermie donnerait également des résultats positifs. Un antibiogramme est nécessaire afin d'éviter l'utilisation de molécules inefficaces.

\section{La prise en charge}

Elle doit être précoce. Une hospitalisation est indispensable chez l'adulte en cas de frisson ou d'hyperthermie dépassant les $38^{\circ} 5$ ou en présence de facteurs favorisants tels que le diabète et l'infection à VIH. Chez l'enfant, une hospitalisation est toujours indispensable [14].

Le traitement comporte trois volets principaux :

\section{Le traitement médical}

L'antibiothérapie est systématique quel que soit le résultat de l'examen bactériologique. Elle est faite par voie intra-veineuse et adaptée à l'antibiogramme. En l'absence d'indications précises, elle associe 10 millions UI de pénicilline et $1,5 \mathrm{~g}$ de métronidazole par jour chez l'adulte et 50000 à $100000 \mathrm{Ul} / \mathrm{kg} / 24 \mathrm{~h}$ de pénicilline associée à 20$30 \mathrm{mg} / \mathrm{kg} / 24 \mathrm{~h}$ de métronidazole chez l'enfant. L'amoxicilline-acide clavulanique peut être utilisée à la dose de 100 mg/kg/24 h. On passe à l'antibiothérapie per os dès que l'état du patient s'améliore ou après $48 \mathrm{~h}$ d'apyrexie. On a recours 
aux macrolides en cas d'allergie à la pénicilline. La durée du traitement est au minimum 10 jours et peut aller jusqu'à la guérison complète.

Un antalgique est toujours nécessaire, et le paracétamol est le plus utilisé. Une corticothérapie à forte dose et de courte durée peut être prescrite en cas d'œdème obstructif [13-14].

\section{Le traitement chirurgical}

Au stade d'inflammation périapicale aigue, un simple drainage par voie endocanalaire serait suffisant pour arrêter l'évolution. Au stade de collection purulente, une incision de drainage transmuqueuse ou trancutanée est nécessaire. Ce drainage doit concerner toutes les loges de la collection et être associé à un débridement tissulaire. Les éventuels tissus nécrotiques sont excisés. Le système de drainage laissé en place (mèche de gaze ou drain de Delbet) permet en plus un lavage biquotidien si nécessaire (dakin, polyvidone iodée ou eau oxygénée). En cas de

\section{RÉFÉRENCES}

1 - Gehanno P, Moisy N, Guedon, Depondt J, regnier B. Les cellulites cervicales extensives. Congrès de la Société Internationale d'ORL et de chirurgie cervicofaciale des pays francophones. Nantes 1988.

2 - Barnabe D, Briche T, Raynal M. Les cellulites cervicales. Rev Off Soc Fr ORL Chir Cervicofac 2003 ; 77 : 8-12.

3 - Potard G. Cellulites de la face et du cou. Rev Off Soc Fr ORL Chir Cervicofac. 2000 ; 64 : 11-26.

4 - Kpemissi E. Cervico-facial cellulitis of oral and dental origin : study of 26 cases at the Lome University Hospital. 1995 ; 116 : 195-7.

5 - Gehanno P, Saint-Guily JL, Regnier B, Vachon F. Cellulitis of the neck due to anaerobic germs: report of 10 cases. Ann Otolaryngol Chir Cervicofac 1982 ; 99 : 41-6.

6 - Gaillard A. Cellulites et fistules d'origine dentaire. Encycl Méd Chir, Elsevier-Masson, Paris. 1989 ; 22-033 A10.

7 - Coales UF, Tandon P, Hinton AE. Abcès parapharyngé dû à un corps étranger. Lettre ORL Chir Cervicofac $1999 ; 247: 20$.

8 - Dordain ML, Coutant G, Algayres JP, Jancovici R, Pats B, Daly JP. Cellulitis secondary to acute thyroiditis in a patient under corticotherapy. Presse Med 1997 ; $26: 319-20$.

9 - Herman PH, Kici S, Portier F. Les cellulites cervicales extensives. Rapports à la Société Française ORL et Chir Cervico-fac. 2002 : 163-9. diffusion médiastinale, une thoracotomie de drainage est faite sous anesthésie générale [19-20]. La dent causale est à extraire après évaluation.

\section{Une éventuelle réanimation}

Elle est réalisée en cas d'altération de l'état général et/ou de dyspnée obstructive pouvant indiquer une intubation, voire une trachéotomie.

\section{Evolution}

Le pronostic est généralement favorable mais les séquelles sont fréquentes, plus ou moins gênantes : cicatrice disgracieuse, fibrose cicatricielle, troubles fonctionnels... Dans un cas l'évolution a été fatale : il s'agissait d'une cellulite cervicofaciale à point de départ dentaire, avec une prise en charge trop tardive. Le patient, âgé de 57 ans, présentait un état général très altéré avec une dénutrition secondaire à un trismus serré. Le décès est dû à un choc septique associé à une détresse respiratoire.

10 - Verdalle PB, Roguet E, Raynal M. Les cellulites cervicomédiastinales nécrosantes : à propos de 3 cas. Ann Oto Laryngol Chir Cervicofac 1997 ; 114 : 302-9.

11 - Berenbaum F. Non-steroidal anti-inflammatory agents. Rev Prat $2003 ; 53: 502-6$.

12 - Bado F. Cellulites cervicales diffuses : à propos de 15 cas. Rev Stomatol Chir Maxillofac $1997 ; 98: 266$.

13 - Tran BH, Manach Y. Les urgences ORL. Rapport de la Société française. 2002 ; 163-8.

14 - Simon D, Franchi G, Ferrads JY. Cellulite faciale d'origine dentaire. Med Express ESTM 2000 : 46-47.

15 - Auboyer C, Charier D, Jospe R. Cellulites, fasciites, myosites, gangrène gazeuse. Encycl Med Chir, ElsevierMasson, Paris, 2001,36 $983 \mathrm{H} 10$.

16 - Wattel F, Mathieu D, Bocquillon N. Infections anaérobies des tissus mous (pp 256-87). Traité de médecine hyperbare. Wattel F et Mathieu D eds, Ellipses, Paris.

17 - Mohammedi I. Fasciite nécrosante cervicale rélévant une infection à VIH. Ann Oto Laryngol Chir Cervicofac. 1997 ; $114: 228-30$.

18 - Derancourt C. Management necrotizing cellulitis and fasciitis. Med Infect 2000 ; 30 : 420-6.

19 - Nguyen DC. Prise en charge des médiastinites et des abcès médiastinaux : à propos de 56 observations. Ann Chir $2003 ; 128: 570-1$.

20 - Chat I, Bouklata S, Chellaoui M, Benamour-Ammar. Médiastinite aiguë non traumatique. Arch Ped 2002 ; $9: 385-7$. \begin{tabular}{l}
$\begin{array}{l}\text { médecine } \\
\text { buccale } \\
\text { chirurgie } \\
\text { buccale }\end{array}$ \\
\hline VOL. 14, N $N^{\circ} 1$ \\
2008
\end{tabular}

page 39 